\title{
Inter-predictability of Neuroprognostic Modalities After Cardiac Arrest
}

Tapan Kavi ${ }^{1}$, Masoom Desai ${ }^{2}$, Furkan M. Yilmaz ${ }^{1}$, Bhavika Kakadia ${ }^{1}$, Evren BurakgaziDalkilic $^{1}$, Gentle S. Shrestha ${ }^{3}$

1. Neurology, Cooper Neurological Institute, Cooper University Hospital, Camden, USA 2. Neurology, Northwestern University Feinberg School of Medicine, Chicago, USA 3. Critical Care, Tribhuvan University Teaching Hospital, Institute of Medicine, Kathmandu, NPL

$\square$ Corresponding author: Furkan M. Yilmaz, mdfurkanyilmaz@yahoo.com Disclosures can be found in Additional Information at the end of the article

\section{Abstract}

\section{Introduction}

At present, there is an emphasis on a multi-modal approach to neuro-prognostication after cardiac arrest using clinical examination, neurophysiologic testing, laboratory biomarkers, and radiological studies. However, this necessitates significant resource utilization and can be challenging in under-resourced clinical settings. Hence, we sought to determine the interpredictability and correlation of prognostic tests performed in patients after cardiac arrest.

\section{Methods}

Fifty patients were included through neurophysiology laboratory data for this retrospective study. Clinical, radiological and neurophysiological data were collected. Neurophysiological data were re-evaluated by a board-certified neurophysiologist for the purpose of the study. Chisquare testing was used to evaluate the correlation between different diagnostic modalities.

\section{Results}

We found that a non-reactive electroencephalogram (EEG) had a predictive value of $79 \%$ for absent bilateral cortical responses (N20) with somatosensory evoked potentials (SSEP). On the other hand, absent bilateral cortical responses N20 had 87\% predictive value for a non-reactive EEG. Also, absent cortical responses and non-reactive EEG had predictive values of $78 \%$ and $72 \%$ for anoxic injury on magnetic resonance imaging (MRI) brain respectively with a nonsignificant difference on chi-square testing. Individually, absent bilateral N20 SSEP, a nonreactive EEG and anoxic brain injury on MRI studies were highly predictive of poor outcome [modified Rankin scale $(\mathrm{mRS})>4$ ] at hospital discharge.

Received 03/18/2019

Review began 04/08/2019 Review ended 04/10/2019 Published 04/17/2019

\section{(c) Copyright 2019}

Kavi et al. This is an open access article distributed under the terms of the Creative Commons Attribution License CC-BY 3.0., which permits unrestricted use, distribution, and reproduction in any medium, provided the original author and source are credited.

\section{Conclusion}

Neuroprognostication in a post-cardiac arrest setting is often limited by self-fulfilling prophecy. Given the lack of absolute correlation between different modalities used in postcardiac arrest patients, the value of the multi-modal approach to neuro-prognostication is highlighted by this study.

Categories: Neurology

Keywords: prognostication, cardiac arrest, eeg, ssep, multi-modal approach 


\section{Introduction}

Neuro-prognostication in patients with an anoxic brain injury after cardiac arrest is challenging, especially with the advent of therapeutic hypothermia (TH) or targeted temperature management (TTM). Current recommendations are to use a multi-modal approach of clinical examination, neurophysiologic testing, biomarkers, and radiological studies [1-3]. Specific results with each of these modalities have been correlated with outcomes in patients with post-anoxic injury and assist in prognosticating patients post-cardiac arrest [1-3]. However, there are no reported studies to our knowledge evaluating the correlation of different modalities used in patients post-cardiac arrest. Electroencephalogram (EEG) and somatosensory evoked potential (SSEP) have been established as robust prognostic markers in cardiac arrest [3]. Non-reactivity to a standard nociceptive, auditory, and visual stimuli and absent N20 SSEP responses post rewarming have been associated with poor neurologic outcomes in post-cardiac arrest patients. In addition, patients with severe anoxic injury on neuro-imaging specifically magnetic resonance imaging have been associated with poor outcomes. Hence, we conducted this study to assess the correlation between different prognostic modalities used in cardiac arrest. We studied if one prognostic modality could predict the results for another modality used in patients post-cardiac arrest. We also correlated the findings obtained on different prognostic modalities with outcomes in these patients. If we could establish a good prediction of outcome with different modalities based on one particular modality, it can help that would spare extensive resource utilization in patients post-cardiac arrest.

\section{Materials And Methods}

Patients were identified by retrospective chart review process of the electronic medical record and neurophysiology laboratory studies. A total of 55 successive SSEPs performed in patients with a suspected anoxic brain injury after cardiac arrests were identified between October 2016 and September 2018. Five patients were excluded because of incomplete data. Data collection included patient characteristics such as age, gender, initial cardiac rhythm, initial Glasgow coma scale (GCS). In addition, neuro-prognostic markers such as EEG and SSEP results, magnetic resonance imaging (MRI) brain results, neurological exam, and data on targeted temperature for these patients were obtained. Restricted diffusion or hyperintensity with FLAIR (fluid-attenuated inversion recovery) sequences involving cerebral cortex, or deep nuclei in basal ganglia was used to identify anoxic brain injury on MRI brain. Also, outcome measures such as modified Rankin scale (mRS) at discharge and mortality were elucidated. There was a re-evaluation of previously performed EEG and SSEP studies by a board-certified neurophysiologist for the purpose of this study. Chi-square testing was used as the statistical method to assess the correlation between different diagnostic modalities.

This retrospective study was performed after approval from our Institutional Review Board. Being a retrospective study, no approval from ethical standards committee, institutional or licensing committee, or informed consent from participants was obtained.

\section{Results}

We included 55 patients in the study. Five patients were excluded due to limitations in extracting study results. Majority of the patients, 48 (96\%), were subjected to targeted temperature management (TTM) with a goal temperature of 36 degrees Celsius as per institutional practice, as seen in Table 1. At our institution, patients qualify for TTM if their examination after cardiac arrest reveals a GCS score of $\leqslant 8$ (in accordance with the TTM trial) [4], and presentation is within six hours of cardiac arrest. The remaining patients did not undergo TTM due to presentation outside the window for cooling. 


\section{Cureus}

\section{Number}

Mean age (years)

Male \%

Initial shockable rhythm percent

Percent patients receiving $\operatorname{TTM}^{\dagger}$

\section{TABLE 1: Baseline characteristics}

*: Ventricular tachycardia or ventricular fibrillation are shockable rhythms.

†: TTM - Targeted temperature management with target of 36 degrees Celsius.

The median initial GCS score for the patients in our study was 4, as seen in Table 2, with a median motor score of 2. Median GCS score at 72 hours after cardiac arrest improved to 5, and median motor score to 3 . About half the patients had pupillary and corneal reactivity at presentation, which increased to 36 (72\%) and 33 (66\%) for pupillary and corneal reactivity respectively at 72 hours after cardiac arrest. We use a multimodal approach for neuroprognostication at our institution and prognosticate no earlier than 72 hours after return of spontaneous circulation (ROSC). So, all these patients had SSEP, EEG, and MRI brain performed as part of neuro-prognostication along with the neurological examination.

\section{Median Initial GCS}

Median GCS at 72 hours post CA

Median GCS motor score at presentation

Median GCS motor score at 72 hours

Pupillary Reactivity at presentation

Pupillary reactivity at 72 hours

Corneal Reflex presence at presentation

Corneal Reflex presence at 72 hours

Mortality
4

5

2

3

$56 \%$

$72 \%$

$48 \%$

$66 \%$

$74 \%$

\section{TABLE 2: Clinical parameters}

GCS: Glasgow coma scale; CA: Cardiac arrest. 


\section{Cureus}

stimulation of bilateral median nerves during SSEP evaluation. As per institutional protocol, continuous EEG is obtained on all cardiac arrest patients during the rewarming phase of TTM, unless indicated earlier (to rule out myoclonic status epilepticus or non-convulsive status epilepticus). Rewarming is conducted at a rate of 0.25 degree Celsius rise per hour at our institution. For the patients included in this study, EEG was performed at a median of 38 hours from cardiac arrest. In addition, SSEPs were performed at a median of 84 hours after cardiac arrest (48 hours after rewarming completion). The correlation of results between these two closely related neurophysiologic tests was assessed using a 2 × 2 table as seen in Table 3 . We found that a non-reactive EEG (to standard nociceptive, auditory, and visual stimuli) was not always predictive of absent cortical responses (N20) on SSEP with a predictive value of 79\%. Correspondingly, absent cortical responses on SSEP were predictive of non-reactivity on EEG in $87 \%$ of the cases. The predictive value of good prognostic sign on SSEP (intact cortical response) for good prognostic sign on EEG (reactive EEG) was very poor, with a predictive value of $18 \%$. This suggests that both these tests have better inter-predictability for poor prognostic signs than good prognostic signs.

\begin{tabular}{|c|c|c|c|}
\hline & Reactive EEG & Non-reactive EEG & PV \\
\hline SSEP- N20 present & 2 & 9 & 2/11 (18\%) \\
\hline SSEP- N20 absent & 5 & 34 & $34 / 39(87 \%)$ \\
\hline
\end{tabular}

\section{TABLE 3: SSEP and EEG inter-predictability}

SSEP-N20: Somatosensory evoked potential N20; EEG: Electroencephalography; PV: Predictive value.

We also assessed the predictability of SSEP and EEG poor prognostic findings for evidence of anoxic brain injury on neuroimaging studies. As shown in Table 4, absent cortical responses and non-reactive EEG had predictive values of $85 \%$ and $81 \%$ for anoxic injury on neuroimaging respectively with a non-significant difference between the two tests (EEG and SSEP) to predict anoxic injury on chi-square testing. Moreover, the correlation of good prognostic signs (present cortical responses, reactive EEG and absence of anoxic injury on neuroimaging) amongst each other was very poor in this study population. 


\section{Cureus}

\begin{tabular}{|c|c|c|c|c|c|c|c|}
\hline & $\begin{array}{l}\text { MRI- anoxic } \\
\text { injury absent }\end{array}$ & $\begin{array}{l}\text { MRI- anoxic } \\
\text { injury present }\end{array}$ & PV & & $\begin{array}{l}\text { MRI- anoxic } \\
\text { injury absent }\end{array}$ & $\begin{array}{l}\text { MRI- anoxic } \\
\text { injury present }\end{array}$ & PV \\
\hline $\begin{array}{l}\text { SSEP- N20 } \\
\text { present }\end{array}$ & 4 & 7 & $\begin{array}{l}4 / 11 \\
(36 \%)\end{array}$ & $\begin{array}{l}\text { EEG- } \\
\text { reactive }\end{array}$ & 2 & 5 & $\begin{array}{l}2 / 7 \\
(29 \%)\end{array}$ \\
\hline $\begin{array}{l}\text { SSEP- N20 } \\
\text { absent }\end{array}$ & 6 & 33 & $\begin{array}{l}33 / 39 \\
(85 \%)\end{array}$ & $\begin{array}{l}\text { EEG non- } \\
\text { reactive }\end{array}$ & 8 & 35 & $\begin{array}{l}35 / 43 \\
(81 \%)\end{array}$ \\
\hline
\end{tabular}

\section{TABLE 4: SSEP, EEG and neuroimaging inter-predictability}

SSEP-N20: Somatosensory evoked potential N20; MRI: Magnetic resonance imaging; EEG: Electroencephalography; PV: Predictive value.

We assessed the predictability of SSEP, neuroimaging, and EEG for outcomes using the modified Rankin Scale at hospital discharge. A cut-off of mRS 4 at hospital discharge was used to consider a good outcome. As seen in Table 5, absent cortical responses (100\%) and non-reactive EEG (100\%) had very high predictive value for poor outcome (mRS > 4). Evidence of anoxic injury on MRI brain also had a very high predictive value for poor outcome (97\%). Moreover, the predictive value of SSEP, EEG or MRI good prognostic signs for a good outcome was very poor; $27 \%, 43 \%$, and $20 \%$ respectively in this study population. The mortality rate in our study was high at 37/50 (74\%), which reflects a high prevalence of severe anoxic brain injury in the study population.

\begin{tabular}{|c|c|c|c|c|c|c|c|c|c|c|c|}
\hline & $\begin{array}{l}\mathrm{mRS}< \\
\text { or }=4\end{array}$ & $\begin{array}{l}\mathrm{mRS} \\
>4\end{array}$ & PV & & $\begin{array}{l}\mathrm{mRS}< \\
\text { or }=4\end{array}$ & $\begin{array}{l}\mathrm{mRS} \\
>4\end{array}$ & PV & & $\begin{array}{l}\mathrm{mRS}< \\
\text { or }=4\end{array}$ & $\begin{array}{l}\mathrm{mRS} \\
>4\end{array}$ & PV \\
\hline $\begin{array}{l}\text { SSEP-N20 } \\
\text { present }\end{array}$ & 3 & 8 & $\begin{array}{l}3 / 11 \\
(27 \%)\end{array}$ & $\begin{array}{l}\text { MRI- anoxic } \\
\text { injury absent }\end{array}$ & 2 & 8 & $\begin{array}{l}2 / 10 \\
(20 \%)\end{array}$ & $\begin{array}{l}\text { EEG } \\
\text { reactive }\end{array}$ & 3 & 4 & $\begin{array}{l}3 / 7 \\
(43 \%)\end{array}$ \\
\hline $\begin{array}{l}\text { SSEP- } \\
\text { N20 } \\
\text { absent }\end{array}$ & 0 & 39 & $\begin{array}{l}39 / 39 \\
(100 \%)\end{array}$ & $\begin{array}{l}\text { MRI- anoxic } \\
\text { injury present }\end{array}$ & 1 & 39 & $\begin{array}{l}39 / 40 \\
(97 \%)\end{array}$ & $\begin{array}{l}\text { EEG non- } \\
\text { reactive }\end{array}$ & 0 & 43 & $\begin{array}{l}43 / 43 \\
(100 \%)\end{array}$ \\
\hline
\end{tabular}

\section{TABLE 5: SSEP, EEG, MRI and outcome prediction}

SSEP-N20: Somatosensory evoked potential N20; mRS: Modified Rankin Scale; MRI: Magnetic resonance imaging; EEG: Electroencephalography; PV: Predictive value.

The preliminary results of this study were presented at the Neurocritical Care Society (NCS) 16th Annual Meeting in 2018. (Abstract: Yilmaz FM, Kakadia B, Kouch M, Hunter K, BurakgaziDalkilic E, Kavi T: Inter-predictability of Neuroprognostic Modalities after Cardiac Arrest. Presented at the Neurocritical Care Society (NCS) 16th Annual Meeting, Boca Raton, FL, USA; September 25-28, 2018.)

\section{Discussion}


Neurologic prognostication after cardiac arrest is a crucial part of the management of patients post-cardiac arrest with significant consequences. Modulation of brain injury and slowed metabolism of sedative medications leads to delayed recovery of brain function and findings on neurologic examination [5]. For this reason, neurological exam and prognostication early on after resuscitation from cardiac arrest can be unreliable and waiting at least 72 hours after ROSC is recommended [1-3]. Recommendations also include using a multimodal approach to neurologic prognostication by using clinical examination, neurophysiological testing, radiological testing and laboratory biomarkers [1-3]. The ideal neuro-prognostic method or tool should have a negligible false positive rate (FPR) and should approach zero. This would ensure avoidance of premature discontinuation of life-supportive measures for patients who have a chance of meaningful recovery in the future.

In the setting of therapeutic hypothermia, the clinical examination remains a robust tool for neuro-prognostication. The absence of bilateral pupillary reflex at 72 hours after cardiac arrest has an FPR of $0.5 \%$ for prediction of poor neurologic outcome [6-9]. On the other hand, corneal reflexes, motor response to pain and early post-anoxic myoclonus have a higher FPR and lower utility for neuro-prognostication [6-12]. When malignant features (burst suppression pattern, myoclonic status epilepticus) on the EEG findings are seen along with myoclonus, the predictability for poor prognosis is much higher [13]. Neurophysiologic testing with EEG and SSEP has retained high utility for prognostication. EEG abnormalities such as suppressed or discontinuous background, and burst suppression have an FPR close to zero for prognosis, but the absence of reactivity or epileptiform discharges have higher FPR [14-16]. In addition, bilateral absence of cortical responses on SSEP testing has been shown to have FPR close to zero for neuro-prognostication in this patient cohort [17]. Computed tomography (CT) and MRI have less data on prognostication as compared to clinical or neurophysiologic testing, however loss of gray-white differentiation on CT is a very reliable predictor (FPR of zero in multiple studies) $[18,19]$. Serum Neuron-Specific Enolase has lower utility for prognostication after TTM, as even higher cut-offs have been reported with good neurologic outcomes [20-22].

However, performing all these prognostic studies can lead to considerable resource utilization and imposes a significant financial burden in a resource-limited clinical setting. If studies have a high correlation for poor prognostic markers, selected tests could be utilized for prognostication. SSEP and EEG, being closely related neurophysiological tests, are particularly interesting in this regard. SSEP differs from EEG in that it is an evaluation of afferent response, while EEG is an evaluation of efferent response-generation of neuronal signals. EEG reactivity in comatose survivors of CA seems to be a stronger predictor of good prognosis than the presence of cortical responses on SSEP testing [23].

Our study found that there was not an absolute correlation between markers of poor outcome when assessed by using SSEP, EEG, and MRI. The reason for this can be explained by the fact that each of these modalities has specific limitations. For example, reactivity on the EEG as a prognostic marker can be limited by the applied stimulus, the inter-rater reliability of reactivity on EEG and at times confounding factors like sedation usage in this patient population. SSEPs, while a robust tool for prognostication, are still not absolute markers of poor outcomes and carry limitations of being operator dependent. Neuro-imaging for evaluation of anoxic brain injury has limited prognostic value by itself in this patient cohort. So, this study re-affirms the value of multi-modal approach in neuro-prognostication of patients post-cardiac arrest. The study also confirms previous findings that absent cortical responses on SSEP are a robust tool for neuro-prognostication in patients post-cardiac arrest.

Our study has certain limitations. One of the main limitations of this study is the withdrawal of life support, reflecting the self-fulfilling prophecy of the intensivists taking care of these patients. The small sample size is also a limitation of the study. Also, the study population is biased towards those with severe anoxic brain injury. SSEP and other prognostic tests were 
obtained in those with a poor neurologic examination after cardiac arrest, and hence the predictive value seen in this study is limited in this population and not applicable to all survivors of cardiac arrest. This can also be the reason for the poor predictive value of these tests for a good prognosis.

It would be interesting to assess if a model based on a combination of the different diagnostic modalities and clinical examination predicts outcomes post-cardiac arrest more robustly than a single diagnostic study by itself.

\section{Conclusions}

The study shows that there is not an absolute correlation between different prognostic markers when it comes to predicting poor outcomes. Given the limitations associated with withdrawal of life support in neuro-prognostication after cardiac arrest and the lack of absolute predictability of one test for the other, the study reaffirms the value of a multi-modal approach to prognostication after cardiac arrest.

\section{Additional Information \\ Disclosures}

Human subjects: Consent was obtained by all participants in this study. Cooper University Hospital Institutional Review Board issued approval 17-161EX. Animal subjects: All authors have confirmed that this study did not involve animal subjects or tissue. Conflicts of interest: In compliance with the ICMJE uniform disclosure form, all authors declare the following: Payment/services info: All authors have declared that no financial support was received from any organization for the submitted work. Financial relationships: All authors have declared that they have no financial relationships at present or within the previous three years with any organizations that might have an interest in the submitted work. Other relationships: All authors have declared that there are no other relationships or activities that could appear to have influenced the submitted work.

\section{References}

1. Sandroni C, Geocadin RG: Neurological prognostication after cardiac arrest. Curr Opin Crit Care. 2015, 21:209-214. 10.1097\%2FMCC.0000000000000202

2. Golan E, Barrett K, Alali AS, et al.: Predicting neurologic outcome after targeted temperature management for cardiac arrest: systematic review and meta-analysis. Crit Care Med. 2014, 42:1919-1930. 10.1097/CCM.0000000000000335

3. Oddo M, Rossetti AO: Early multimodal outcome prediction after cardiac arrest in patients treated with hypothermia. Crit Care Med. 2014, 42:1340-1347. 10.1097/CCM.0000000000000211

4. Nielsen N, Wetterslev J, Cronberg T, et al.: Targeted temperature management at $33^{\circ} \mathrm{C}$ versus $36^{\circ} \mathrm{C}$ after cardiac arrest. N Engl J Med. 2013, 369:2197-2206. 10.1056/NEJMoa1310519

5. Grossestreuer AV, Abella BS, Leary M, et al.: Time to awakening and neurologic outcome in therapeutic hypothermia-treated cardiac arrest patients. Resuscitation. 2013, 84:1741-1746. 10.1016/j.resuscitation.2013.07.009

6. Rossetti AO, Oddo M, Logroscino G, Kaplan PW: Prognostication after cardiac arrest and hypothermia: a prospective study. Ann Neurol. 2010, 67:301-307. 10.1002/ana.21984

7. Greer DM, Yang J, Scripko PD, et al.: Clinical examination for prognostication in comatose cardiac arrest patients. Resuscitation. 2013, 84:1546-1551.

10.1016/j.resuscitation.2013.07.028

8. Sandroni C, Cariou A, Cavallaro F, et al.: Prognostication in comatose survivors of cardiac arrest: an advisory statement from the European Resuscitation Council and the European Society of Intensive Care Medicine. Intensive Care Med. 2014, 40:1816-1831. 10.1007/s00134014-3470-x 
9. Wijdicks EF, Hijdra A, Young GB, Bassetti CL, Wiebe S: Practice parameter: prediction of outcome in comatose survivors after cardiopulmonary resuscitation (an evidence-based review): report of the Quality Standards Subcommittee of the American Academy of Neurology. Neurology. 2006, 67:203-210. 10.1212/01.wnl.0000227183.21314.cd

10. Bouwes A, Binnekade JM, Kuiper MA, et al.: Prognosis of coma after therapeutic hypothermia: a prospective cohort study. Ann Neurol. 2012, 71:206-212. 10.1002/ana.22632

11. Lucas JM, Cocchi MN, Salciccioli J, et al.: Neurologic recovery after therapeutic hypothermia in patients with post-cardiac arrest myoclonus. Resuscitation. 2012, 83:265-269. 10.1016/j.resuscitation.2011.09.017

12. Seder DB, Sunde K, Rubertsson S, et al.: Neurologic outcomes and postresuscitation care of patients with myoclonus following cardiac arrest. Crit Care Med. 2015, 43:965-972. 10.1097/CCM.0000000000000880

13. Bouwes A, van Poppelen D, Koelman JH, et al.: Acute posthypoxic myoclonus after cardiopulmonary resuscitation. BMC Neurol. 2012, 12:63. 10.1186/1471-2377-12-63

14. Fugate JE, Wijdicks EF, Mandrekar J, et al.: Predictors of neurologic outcome in hypothermia after cardiac arrest. Ann Neurol. 2010, 68:907-914. 10.1002/ana.22133

15. Sivaraju A, Gilmore EJ, Wira CR, et al.: Prognostication of post-cardiac arrest coma: early clinical and electroencephalographic predictors of outcome. Intensive Care Med. 2015, 41:1264-1272. 10.1007/s00134-015-3834-X

16. Cloostermans MC, van Meulen FB, Eertman CJ, Hom HW, van Putten MJ: Continuous electroencephalography monitoring for early prediction of neurological outcome in postanoxic patients after cardiac arrest: a prospective cohort study. Crit Care Med. 2012, 40:2867-2875. 10.1097/CCM.0b013e31825b94f0

17. Leithner C, Ploner CJ, Hasper D, Storm C: Does hypothermia influence the predictive value of bilateral absent N20 after cardiac arrest?. Neurology. 2010, 74:965-969. 10.1212/WNL.0b013e3181d5a631

18. Cristia C, Ho ML, Levy S, et al.: The association between a quantitative computed tomography (CT) measurement of cerebral edema and outcomes in post-cardiac arrest-a validation study. Resuscitation. 2014, 85:1348-1353. 10.1016/j.resuscitation.2014.05.022

19. Lee BK, Jeung KW, Song KH, et al.: Prognostic values of gray matter to white matter ratios on early brain computed tomography in adult comatose patients after out-of-hospital cardiac arrest of cardiac etiology. Resuscitation. 2015, 96:46-52. 10.1016/j.resuscitation.2015.07.027

20. Daubin C, Quentin C, Allouche S, et al.: Serum neuron-specific enolase as predictor of outcome in comatose cardiac-arrest survivors: a prospective cohort study. BMC Cardiovasc Disord. 2011, 11:48. 10.1186/1471-2261-11-48

21. Stammet P, Collignon O, Hassager C, et al.: Neuron-specific enolase as a predictor of death or poor neurological outcome after out-of-hospital cardiac arrest and targeted temperature management at 33 degrees C and 36 degrees C. J Am Coll Cardiol. 2015, 65:2104-2114. 10.1016/j.jacc.2015.03.538

22. Wiberg S, Hassager C, Stammet P, et al.: Single versus serial measurements of neuron-specific enolase and prediction of poor neurological outcome in persistently unconscious patients after out-of-hospital cardiac arrest - a TTM-trial substudy. PLoS ONE. 2017, 12:e0168894. 10.1371/journal.pone.0168894

23. Oddo M, Rossetti AO: Predicting neurological outcome after cardiac arrest . Curr Opin Crit Care. 2011, 17:254-259. 10.1097/MCC.0b013e328344f2ae 